\title{
CORRESPONDENCE
}

\section{How government spending cuts put lives at risk}

World leaders currently making tough economic decisions should be guided by the physicians' mantra: "First, do no harm". Austerity programmes, even if justifiable in terms of promoting growth (itself highly questionable), may exacerbate the health risks posed by financial crises.

Inflicting short-term pain constitutes a massive, uncontrolled experiment with people's lives. Recessions themselves pose a risk to health, but empirical data reveal that the decisions made by governments crucially determine how bad the outcome will be.

To mitigate the effects of the Great Depression in the 1930s, US policy-makers created a socialwelfare system and invested in public-health programmes. Mortality rates fell by about $10 \%$, mainly owing to a decrease in infectious diseases and roadtraffic accidents (P. Fishback et al. Rev. Econ. Stat. 89, 1-14; 2007).

But in several eastern European countries after the economic collapse of the early 1990s, policymakers massively cut social and health budgets, while deregulating the economy. Mortality rates rose by about $40 \%$, mainly through heart attack, stroke, alcoholrelated death, suicide and homicide. There were more than 3 million excess deaths (D. S. et al. Lancet 373, 399-407; 2009) the worst peacetime mortality crisis in the past half-century.

European Union (EU) mortality trends during recessions in the past three decades indicate that member states can avoid a rise in suicide rates by spending US $\$ 200$ per capita a year or more on labour-market programmes, designed to improve people's chances of gaining employment (D. S. et al. Lancet 374, 315-323; 2009). In those spending less than $\$ 70$ - such as Spain and the mainly eastern European countries that joined from 2004 onwards - a deteriorating economy correlates with a rise in suicide rate. But in Finland and Sweden, which spend at least $\$ 300$, economic change has no discernible short-term effect on overall population health.

What can scientists do to help protect public health in times of economic crisis? They should promote an evidence-based approach to economic and publichealth recovery, analysing past successes and failures. This will lead to a better understanding of why some people, households, communities and societies are resilient to external shocks.

David Stuckler Department of Sociology, Oxford University, Oxford OX11DP, UK

e-mail: david.stuckler@chch.ox.ac.uk Sanjay Basu Department of Medicine, University of California, San Francisco, California, USA

Martin McKee London School of Hygiene and Tropical Medicine, London, UK

\section{Biodiversity: linking Singapore's fragmented habitats}

As we celebrate the International Day for Biological Diversity on 22 May, Singapore - a participant in the preparatory committee of the 1992 United Nations Earth Summit and a signatory to the Convention on Biological Diversity - is making another contribution.

In addition to its proposed index on cities' biodiversity, to measure urban efforts towards conservation and sustainable development (Nature 460, 33; 2009), Singapore is to construct an ecological corridor known as the Eco-Link over the Bukit Timah expressway. With completion expected by 2013, this hourglassshaped bridge will re-establish a connection that was severed in 1985 between the city's Bukit Timah and Central Catchment nature reserves.

The corridor is intended for tropical conservation and should redress trade-offs made in the past for economic reasons. It has been compared with Natuurbrug Zanderij Crailo ('Crailo sandquarry nature bridge') in the Netherlands. However, its tropical setting is likely to pose engineering and restoration difficulties, owing to the diversity of tropical habitats and inhabitants.

It will be planted up like a forest to enable animal and plant transfer between the two reserves. Longer term, it is hoped that the corridor will restore the ecological balance in the fragmented habitats and rectify the loss of biodiversity. If successful, it could be replicated in other tropical cities to help conserve native biodiversity and to teach us how to restore degraded natural landscapes.

It is instructive that a nation as small, land-scarce, resource-poor and highly urbanized as Singapore is leading this promising initiative.

Kwek Yan Chong, Alex Thiam Koon

Yee, Chow Khoon Yeo

Plant Systematics Laboratory, National University of Singapore, 14 Science

Drive 4, Singapore 117543

e-mail: kwek@nus.edu.sg

\section{Biodiversity: need for balanced reports of solutions and failures}

Some leading conservation biologists deliver unnecessarily gloomy addresses, closing with no solutions or a few anecdotal

success stories. Non-governmental organizations (NGOs), by contrast, can be too optimistic in their fundraising lectures and magazines: false starts and dead ends don't figure, because donors prefer winners. Both groups are misjudging their audiences.

Without the attraction of carefully considered general solutions, young scientists will become disheartened or even dismissive of the conservation crisis. On the other hand, without honest admission that conservation programmes can be messy and sometimes fail, NGOs will not gain the confidence of increasingly well-educated donors. Comparative evaluations do exist of what works in conservation and what doesn't, and they need airing.

Students and philanthropists want to help. But we should strive for a nuanced balance of optimism and brutal honesty in conservation public relations.

Tim Caro Department of Wildlife, Fish and Conservation Biology, University of California, 1Shields Avenue, Davis, California 95616, USA e-mail: tmcaro@ucdavis.edu

\section{Controls needed to reduce problem of plastic contamination}

Your online News report about assay contamination by chemicals leaching from plastic containers (go.nature.com/R7eAFN) doesn't do justice to the scale of the problem and the effort needed to tackle it. The cost of biological experiments performed in standard polypropylene test tubes and polystyrene Petri dishes is enormous, including the price of millions of animals and expensive growth factors. Worse, systematic errors resulting from plastic leaching are biasing conclusions drawn from these experiments.

This could have important implications in stem-cell culture, for example, aggravated by the smooth surface of the Petri dish, which is unlike the cells' natural environment.

Companies should address the problem with innovative products. But many reagents extract additives from plastics, and no manufacturer or legislator can address the whole variety of conditions used in laboratories around the world. Rigorously designing appropriate controls could be another way forward.

Andrei P. Sommer Institute of Micro and Nanomaterials, University of Ulm, 89081 Ulm, Germany e-mail: andrei.sommer@uni-ulm.de Noah Lotan Department of Biomedical Engineering, Technion, 32000 Haifa, Israel 